\title{
Linx
}

Revue des linguistes de l'université Paris X Nanterre

42 | 2000

Approches sociolinguistiques du plan phonique

\section{Absence de repères régionaux et relâchement de la prononciation}

Armstrong Nigel et Boughton Zoë

\section{OpenEdition}

\section{Journals}

Édition électronique

URL : http://journals.openedition.org/linx/794

DOI : $10.4000 /$ linx.794

ISSN : 2118-9692

Éditeur

Presses universitaires de Paris Nanterre

\section{Édition imprimée}

Date de publication : 1 juin 2000

Pagination : 59-71

ISSN : 0246-8743

\section{Référence électronique}

Armstrong Nigel et Boughton Zoë, «Absence de repères régionaux et relâchement de la

prononciation », Linx [En ligne], 42 | 2000, mis en ligne le 22 juin 2012, consulté le 20 avril 2019. URL

http://journals.openedition.org/linx/794 ; DOI : 10.4000/linx.794

Ce document a été généré automatiquement le 20 avril 2019.

Département de Sciences du langage, Université Paris Ouest 


\title{
Absence de repères régionaux et relâchement de la prononciation
}

\author{
Armstrong Nigel et Boughton Zoë
}

\section{0 . Introduction}

1 Dans cet article, nous rendons compte de deux séries de résultats provenant d'une enquête portant sur la variation phonologique en « français standard » : ici, il s'agira des variétés urbaines septentrionnales du français, présentes dans toute couche sociale en France au nord de la Garonne et du Massif central, abstraction faite de régions périphériques telles l'Alsace, la Bretagne occidentale et l'agglomération Lille-Roubaix. Concernant la dimension stylistique, par "standard» nous n'entendons pas renvoyer à un registre formel ; les données langagières dont nous discuterons ici ont été enregistrées au cours d'interviews sociolinguistiques ayant pour but d'approcher la langue parlée au quotidien, dans un registre " consultatif » (Joos 1961). Défini ainsi, l'objet de notre étude s'aligne de façon approximative sur cette notion de « français au quotidien », sens auquel référera ici le terme simple de « français ».

2 L'objectif de notre enquête est d'analyser quelques-unes des implications d'une proposition que l'on trouve assez fréquemment dans la littérature sociolinguistique sur le français, selon laquelle la prononciation du français serait relativement nivelée ou peutêtre «standardisée ». Cette proposition semble être vraie si l'on compare le français aux autres langues occidentales qui ont fait l'objet de recherches variationnistes : les variétés urbaines de l'anglais, le français du Canada et l'espagnol de l'Amérique latine.

Il paraît plausible qu'un nivellement de la prononciation du français puisse entraîner des difficultés d'identification de la provenance régionale des locuteurs, sur la base du seul accent. Cependant, l'un des axiomes de la sociolinguistique propose que tout locuteur se situe, par ses variations langagières, dans un "espace [social] multidimensionnel» (Hudson 1996: 207). Les dimensions principales de cet espace seraient les variables sociales de l'âge, du sexe, de la classe sociale et l'appartenance régionale, aussi bien que le 
style du discours. L'un des aspects perceptuels de ce processus d'auto-situation est la tentative d'identification par l'auditeur des attributs sociaux du locuteur à partir d'une analyse de son langage (entre autres données), comme l'illustre l'exemple des « inconnus dans le train» fourni par Trudgill (1995: 1-2). Une démarche ultérieure concerne l'évaluation; l'auditeur formule un jugement normatif sur la base de son identification. On observe ce processus sous une forme des plus frappantes lors d'une première rencontre, lorsque l'auditeur est obligé d'amorcer ce processus en l'absence d'autres donnés vérifiables concernant le locuteur en question. Ainsi, nous considérons ici les identifications formulées par des auditeurs français sur les traits de prononciation d'un échantillon de locuteurs dont la provenance socio-régionale est inconnue.

Notre hypothèse est que les identifications par les auditeurs de ces traits de prononciation sont susceptibles d'éclairer indirectement la question suivante: dans quelle mesure les variations de prononciation véhiculent-elles des repères permettant de juger des origines socio-régionales des Français? La problématique ultérieure concerne l'importance, pour le locuteur, de la provenance régionale relative aux autres attributs sociaux, notamment la classe sociale.

\section{Les données}

\section{1 Les corpus}

Les données linguistiques, ainsi que celles concernant l'évaluation, que nous examinons ont été collectées en 1997-98 à Nancy et à Rennes. Ces deux villes sont comparables sur le plan linguistique en raison de leur situation dans des régions à sous-strate langue d'oïl, le lorrain roman et le gallo respectivement. Elles sont aussi suffisamment distantes l'une de l'autre pour que tout trait linguistique régional soit localisé dans chaque ville. Les villes sont à peu près à équidistance de Paris : Rennes se trouve à quelques $300 \mathrm{~km}$ de la capitale, Nancy à $275 \mathrm{~km}$ environ. Rennes (pop. 250000 en agglomération) et Nancy (300 000) sont ainsi des centres régionaux possédant des caractéristiques distinctives, tout en étant reliées à la capitale sur les plans commerciaux, administratifs et culturels. Nous faisons ainsi les postulats suivants: les variétés langagières dans chaque ville sont distribuées sur un continuum, relié à la fois à la langue standard et à la langue locale ; ces variétés sont semblables au niveau structurel; et enfin tout locuteur dispose d'une gamme de variétés situées sur ce continuum.

\subsection{Taille et structure des échantillons}

6 Le choix des locuteurs a été déterminé par une combinaison des attributs sociodémographiques de l'âge, du sexe et de la classe sociale. En limitant à quatre le nombre de locuteurs dans chaque groupe, l'échantillon obtenu dans les deux villes est apparu comme suit :

Tableau 1 : Taille et structure des échantillons

\begin{tabular}{|l|l|l|l|l|}
\hline & \multicolumn{2}{|c|}{ Hommes } & \multicolumn{2}{c|}{ Femmes } \\
\hline Classe / Age & CO & CM & CO & CM \\
\hline
\end{tabular}




\begin{tabular}{|l|l|l|l|l|}
\hline 16 à 25 ans & $4 \mathrm{JHO}$ & $4 \mathrm{JHM}$ & $4 \mathrm{JFO}$ & $4 \mathrm{JFM}$ \\
\hline \hline 40 à 60 ans & 4 HAO & 4 HAM & 4 FAO & 4 FAM \\
\hline
\end{tabular}

Légende : JHO = jeune homme, classe ouvrière ; FAM = femme plus âgée, classe moyenne ; etc.

7 Trois styles de discours ont été enregistrés: des interviews ou «conversations provoquées ", des passages à lire et des listes de mots. L'expérience que nous décrivons concerne les interviews.

\subsection{L'épreuve auditive}

8 Les interviews enregistrées dans les deux villes se distinguent principalement par la présence d'une épreuve auditive à la fin de l'interview rennaise. On a demandé à 40 auditeurs rennais d'écouter environ une minute de parole produite par un souséchantillon de huit locuteurs nancéiens. Ce sous-échantillon avait été recopié sur cassette dans un ordre aléatoire. En général, les locuteurs nancéiens avaient été choisis comme représentants de leurs groupes sociaux en fonction de la clarté et de la continuité de la parole produite. Après chaque écoute on a demandé à l'auditeur rennais de répondre à des questions. Nous discutons ici des réponses aux questions suivantes:

A : D'après son accent, la personne appartient-elle à la classe ouvrière ou à la classe moyenne?

Réponses relevées : ouvrière / moyenne / pas de réponse.

B : Pouvez-vous identifier la région d'origine de la personne, d'après son accent? Réponses relevées : la région exacte.

11 L'échantillon d'auditeurs rennais reflète en plus grand la structure de l'échantillon de locuteurs. Faute de place, il n'est pas loisible de discuter des interactions entre les attributs sociaux des auditeurs rennais et leurs réponses aux questions ci-dessus. Harms (1961) a montré que les auditeurs d'origine populaire aussi bien que bourgeoise sont d'une compétence égale quant à leurs estimations de la provenance sociale de locuteurs inconnus.

\subsection{Les réponses à la question $\mathrm{A}$}

Tableau 2 : Les réponses des sujets rennais à la question A

\begin{tabular}{|c|c|}
\hline Classe sociale du sujet nancéien & Accord des auditeurs rennais sur un accent ouvrier (en \%) \\
\hline JHO & 92.5 \\
\hline HAO & 87.5 \\
\hline FAO & 70.0 \\
\hline FAM & 57.5 \\
\hline JFO & 35.0 \\
\hline
\end{tabular}




\begin{tabular}{|c|c|}
\hline JHM & 27.5 \\
\hline JFM & 5.0 \\
\hline HAM & 2.5 \\
\hline
\end{tabular}
connaissance des conclusions de la dialectologie rurale et urbaine, il faut prendre en compte les interactions entre les variables sociales en jeu ici : l'âge, le sexe et la classe sociale. Les résultats que l'on trouve dans la littérature montrent fréquemment que les locuteurs "conservateurs" (plus âgés, féminins, des classes sociales aisées) sont les gardiens des variétés langagières valorisées dans toute communauté. Inversement, ceux qui conservent le vernaculaire sont typiquement masculins et d'origine ouvrière; il se peut que l'âge joue un rôle moins important à cet égard, comme nous le suggérons cidessous. Cette proposition générale produirait une hiérarchie des locuteurs nancéiens (par ordre décroissant de l'accord des auditeurs rennais sur un accent ouvrier) perçu comme suit: HAO ou JHO les plus vernaculaires, et FAM ou HAM les moins localisés (pour le moment nous entendons les termes «vernaculaire» et «localisé» comme coréférentiels; nous verrons ci-dessous que ce n'est pas le cas pour la situation qui nous préoccupe ici). Le tableau 2 confirme grosso modo cette proposition, exception faite notamment de la femme plus âgée de $\mathrm{CM}$, qui était d'origine ouvrière, employée, et qui avait gardé plusieurs traits de prononciation régionaux. Il n'est néanmoins pas certain que l'on puisse s'attendre à ce qu'un ordre homogène se maintienne dans toute communauté linguistique entre les deux pôles de CO et CM. Nous avons indiqué ce domaine d'incertitude dans le tableau en grisé. Il y a incertitude car la différentiation en prononciation est non pas statique, mais dynamique. Ainsi, selon Eckert (1989: 248) :

"Les résultats originaux de Labov [...] ont nettement aligné la classe sociale, le style de discours, les changements en cours, le prestige et les évaluations sur un seul axe. Le continuum hiérarchique socio-économique est en même temps un continuum de changement linguistique, où le degré de changement historique est en corrélation inverse avec le statut socio-économique."

L'image d'un seul axe semble être moins idoine si l'on considère que le prestige est un attribut social multidimensionnel; que les changements linguistiques peuvent intervenir par le bas ou par le haut; et que différents groupes sociaux peuvent promouvoir ces changements. Maintes études récentes ont mis l'accent sur le rôle des femmes dans la promotion des changements linguistiques, et ces résultats s'alignent sur les conclusions des enquêtes diatopiques traditionnelles montrant que les locuteurs conservateurs ruraux sont principalement masculins, plutôt âgés et non mobiles (les "NORMs" de Chambers et Trudgill $1998: 29-30$ ).

Plus récemment, des résultats provenant de l'anglais (J. Milroy et al 1994) ainsi que du français (Hansen 1997, 1998 ; Armstrong et Unsworth 1999) ont montré que les locuteurs masculins urbains, jeunes et plus âgés, ont tendance eux aussi à résister à l'introduction de variantes linguistiques non localisées (et souvent «non standard»), alors que leurs homologues féminins les promeuvent. De même, les femmes utilisent plus fréquemment 
les variables stables, non localisées et «standard ». Peut-être est-il possible de résoudre ce paradoxe en proposant que les femmes promeuvent l'introduction de formes supralocales (et souvent non-standard); sachant que ces formes se trouvent ensuite transformées en des variantes standard en raison même de leur promotion par des locuteurs féminins.

Prenant en compte ces considérations, on peut affirmer qu'un ordre homogène selon l'âge, le sexe et la classe sociale dans toute communauté est difficilement concevable. En particulier, c'est l'ordre intermédiaire entre les pôles mentionnés ci-dessus qui est problématique, car le prestige (soit apparent, soit latent) véhiculé par l'accent varie dans toute communauté en fonction des changements en cours, et en fonction des groupes sociaux qui les effectuent. A en juger par les résultats récents en français que nous examinons ci-dessous, ces groupes seront typiquement composés de locuteurs jeunes, féminins et intermédiaires en termes de classe sociale, et ils seront susceptibles de promouvoir les variantes innovatrices soit par le bas soit par le haut. Vu le nombre de facteurs en jeu et la complexité de leurs interactions, nous soulignons à nouveau que l'ordre que laisse apparaître le tableau 2, abstraction faite de la locutrice FAM, se conforme à peu près à nos attentes. Ainsi pouvons-nous suggérer que les auditeurs rennais ont identifié la provenance sociale des locuteurs nancéiens avec une précision tolérable. Il reste à considérer les traits de prononciation qui ont permis aux auditeurs de le faire (nous examinons cette question en conclusion).

17 Nous traitons maintenant du rapport entre l'accent ouvrier perçu et les réponses des auditeurs rennais à la question $\mathrm{B}$, concernant l'origine régionale perçue des Nancéiens.

\subsection{Les réponses à la question $B$}

Schéma 1 : Rapport entre l'origine ouvrière perçue des locuteurs nancéiens et les réponses exactes à la question $B$

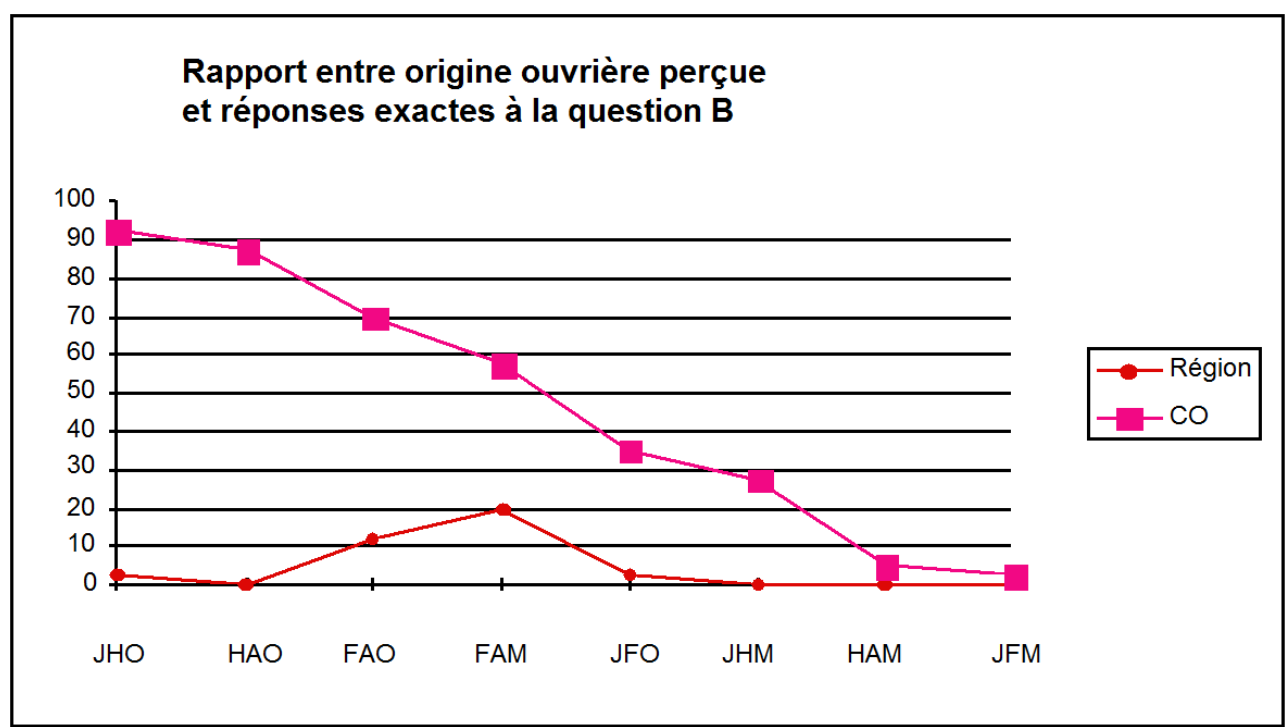

Le schéma 1 montre, juxtaposés avec l'information donnée dans le tableau 2, les pourcentages d'identifications exactes proposés par les auditeurs rennais en réponse à la question B. Il faut remarquer que les réponses «exactes » indiquées dans le schéma 1 résultent d'une interprétation généreuse : ainsi, avons-nous inclus toute réponse portant 
sur le Nord et/ou l'Est de la France. La décision d'exclure ces dernières, et d'inclure seules les réponses qui ont identifié Nancy ou même la Lorraine, se traduirait par des pourcentages de réponses exactes négligeables.

Le schéma 1 montre très clairement que même dans le cas des locuteurs nancéiens identifiés comme possédant un accent ouvrier bien marqué, très peu des auditeurs rennais étaient capables d'identifier leur provenance régionale, même approximativement. Ainsi, le locuteur nancéien JHO, perçu comme tel par 92,5\% des auditeurs, a fait l'objet de $2,5 \%$ de réponses « exactes » (un sur 40 ) concernant son origine régionale. $32,5 \%$ des auditeurs ont hasardé une origine rennaise ou bretonne. Le schéma 1 montre ainsi que le rapport très intime entre accent, classe sociale et origine régionale caractéristique de certains pays comparables est quasiment absent en français, au moins en ce qui concerne cet échantillon de sujets rennais.

\section{Sommaire et discussion}

\subsection{Limites de nos résultats}

Nous faisons remarquer tout d'abord les limites qui nous amènent à prendre des précautions quant aux conclusions que nous formulons: (i) il est question ici de comportements évaluatifs, donc indirects; (ii) les jugements des sujets rennais portent sur huit locuteurs nancéiens seulement; (iii) notre étude concerne uniquement la variété de français définie ci-dessus. Cette variété est importante sur le plan démographique puisqu'elle comprend au moins la moitié de la population française. Plusieurs chercheurs (par exemple Martinet 1945, Walter 1982) ont présenté la variation régionale dans l'ensemble de la France; nous considérons ici une partie importante mais circonscrite du pays.

\subsection{Sommaire des résultats}

21 Nous pouvons résumer les résultats présentés dans le tableau 1 et le schéma 1 comme suit. Dans la présente optique nous écartons la possibilité, pourtant plausible, que les auditeurs rennais aient pu utiliser également des traits autres que phonologiques (la syntaxe, le lexique) pour effectuer leurs identifications.

(i) Le tableau 2 montre un degré de succès élevé de la part des auditeurs rennais dans leurs estimations de la classe sociale des locuteurs nancéiens.

(ii) Le schéma 1 montre un résultat plus intéressant : une grosse disparité entre ce succès d'une part, et le manque de succès des auditeurs rennais dans leurs estimations de la provenance régionale des locuteurs nancéiens d'autre part. Ce résultat contredit les observations faites par des linguistes francophones : par exemple, Walter (1988:159) pose le problème dans les termes suivants: "Un grand banquier parisien parle et surtout prononce différemment de son confrère toulousain ou alsacien, alors que la prononciation de chacun d'entre eux ressemble beaucoup à celle de son employé le plus modeste, originaire de la même région que lui. » Cependant, ces exemples ont le défaut de comparer des cas qui ne sont pas semblables. Dans la mesure où l'on peut faire la comparaison entre nos résultats et les exemples de Walter, les premiers démontrent que les locuteurs de français langue d'oïl sont en effet différenciés sur le plan de la classe 
sociale par leur prononciation, alors que l'élément régional de la différenciation sociale semble avoir été disloqué de ce premier composant social.

\subsection{Discussion : la variation phonologique en français} résultats, les locuteurs-auditeurs français sont capables d'identifier la classe sociale d'un interlocuteur à partir du seul accent, quels traits de prononciation exploitent-ils pour le faire? L'une des réponses indiquées par nos résultats est qu'ils n'utilisent pas les traits linguistiquement arbitraires qui en anglais relient le degré de localisation d'un locuteur à sa classe sociale. Ceci ressort de l'échec notable des sujets rennais à identifier la provenance régionale des Nancéiens. Nous appelons ces variables anglaises arbitraires parce que leur valeur sociale peut varier selon leur localisation, comme le démontre l'exemple de la présence / absence $\mathrm{du} / \mathrm{r} /$. Le /r/ post-vocalique est présent à New York dans le parler des groupes jouissant d'un prestige apparent, alors que la structure inverse prévaut généralement en Grande Bretagne. Ce principe de l'arbitraire des variables est axiomatique en sociolinguistique, mais il se peut que des traits de prononciation plus «naturels » communiquent l'appartenance de classe sociale en France, sans pour autant indiquer l'appartenance régionale, précisément du fait que ces traits sont partagés par toute la communauté linguistique septentrionale. Cette absence de localisation régionale dans les parlers français socialement diagnostiqués est suggérée par le fait que plusieurs chercheurs ont rendu compte de résultats concernant certaines variables dans des espaces géographiquement bien séparés : Tours (Ashby 1988), Paris (Laks 1983) ; Lorraine (Armstrong 1996).

traits naturels » nous entendons les phénomènes d'élision et de neutralisation. Les variables phonétiques du français sujettes à l'élision sont principalement le schwa, le /r/ et le /l/. La neutralisation en français concerne les oppositions vocaliques : les voyelles moyennes, orales ainsi que nasales. Il est évident que l'élision et la neutralisation se produisent dans toute langue, pour des raisons associées aux caractéristiques phonétiques et métriques des segments concernés. Ce qui caractérise le français, c'est que la variation phonologique semble consister principalement en ces phénomènes, à en juger par le fait que la plupart des chercheurs se sont concentrés sur eux seuls. Ceci porte à croire que l'élision et la neutralisation sont effectivement la ressource principale de la phonologie variable dont dispose les locuteurs français.

Il semble ainsi que la variation phonologique arbitraire soit peu importante en français, quoique relativement peu de résultats soient disponibles pour confirmer cette hypothèse. La variation phonologique arbitraire se manifeste surtout dans le système vocalique : l'un des changements de ce type qui semble être en cours est l'antériorisation de la voyelle moyenne postérieure [0], vers la réalisation la plus antérieure [œ], par exemple dans joli réalisé [3œli], alors que la réalisation canonique est [3Jli] (Landick 1995). Un autre phénomène apparemment arbitraire apparu en français septentrional, et peut-être surtout parisien, est la tendance récente vers le "schwa épenthétique", à savoir la réalisation d'un schwa en finale de mot, après une seule consonne phonétique et avant pause, de sorte que c'était Pierre se réalise de façon variable comme [setદpjєs(ə)]. On ne sait pas si la variation entre les /a/ antérieurs et postérieurs continue à véhiculer une signification sociale dans la langue quotidienne, quoiqu'on ait souvent mentionné cette opposition dans la littérature. La neutralisation des voyelles moyennes antérieures 
nasales, distribuée largement en langue d'oïl actuellement, s'explique facilement du point de vue fonctionnel, alors que la variation entre les voyelles moyennes postérieures nasales semble arbitraire dans une optique fonctionnelle (Hansen 1998). En tous les cas, les résultats de Hansen concernent le parler de Parisiens, et la signification sociogéographique de la variation entre les voyelles moyennes postérieures nasales reste incertaine. La liaison variable n'est pas localisée géographiquement en français, quoiqu'on ait observé d'autres structures sociolinguistiques en français du Québec. Il est donc possible que la variation phonologique dans le français «nivelé » relève principalement des phénomènes qui se produisent dans la chaîne de la langue parlée : ce que Gadet (1989 : 71) appelle les « facilités de prononciation ». De ce fait, il semblerait que la variation phonologique en français ne soit pas entièrement «sociolinguistique » dans le sens anglo-saxon que nous entendons ici.

Par conséquent, ces phénomènes suggèrent que la variation phonologique du genre que l'on observe dans l'anglais des Etats-Unis et de la Grande Bretagne a été majoritairement supprimée en français. Les facilités de prononciation - les relâchements - représentent ce qui en reste. Si ces processus sont les éléments principaux de variation phonologique disponibles, les locuteurs de langue d'oïl semblent avoir la possibilité de communiquer les uns aux autres leur identité en termes de classe sociale, mais non pas leur appartenance régionale, ou ceci dans une moindre mesure. Nous proposons maintenant deux conclusions que l'on peut tirer de ces faits.

\section{Conclusions générales}

28 (i) Notre première conclusion concerne la fonction sociale des accents différenciateurs dans les sociétés organisées hiérarchiquement en termes de classe sociale. Si les résultats présentés dans le tableau 2 et le schéma 1 sont représentatifs de la situation du français non méridional, il semble que les Français peuvent identifier la classe sociale d'après l'accent. Ce fait soulève la question plutôt fondamentale de la primauté de cette variable démographique en tant que marqueur de la différenciation sociale dans les communautés linguistiques. Certes, maintes discussions dans la littérature sociolinguistique des concepts tels les «marqueurs » et «indicateurs » ont postulé des interactions entre la différenciation selon la classe et le style de discours (cf. Chambers et Trudgill 1998: 7073). Cet accent mis sur la classe sociale reflète une perception partagée par les sociolinguistes que cette variable serait «la variable indépendante la plus susceptible d'être en corrélation avec l'innovation linguistique » (Chambers et Trudgill $1998: 153$ ). Si l'on considère le contraste entre la situation de l'anglais et du français par rapport à des facteurs historiques, il apparaît que la langue anglaise a évolué de sorte que ses locuteurs possèdent une ressource, le composant régional de l'accent socio-régional, qui ferait défaut aux locuteurs français. Il va de soi que nous voulons éviter tout argument téléologique ; la langue française n'a pas évolué "pour que" les Français puissent retenir la possibilité d'identifier la classe sociale de leurs interlocuteurs, même si cet aspect de l'identité sociale, qui véhicule des données concernant l'accès du locuteur au capital économique et social, représente peut-être l'aspect que les inconnus ont le plus instamment envie d'apprendre lors d'une première rencontre. Nous faisons remarquer en outre que le français semble représenter un contre-exemple à la généralisation, par ailleurs plutôt plausible, avancée par Hudson (1996: 43) selon laquelle « nous employons la prononciation pour identifier nos origines (ou pour laisser entendre nos origines, que 
cela soit vrai ou non) ». Si l'on interprète ici « origine » au sens régional, il est évident que cet attribut social peut être hautement prisé ; il serait alors remarquable que les Français ne jouissent pas des moyens de l'exprimer à travers l'accent.

(ii) La deuxième conclusion, corollaire de la première, concerne l'aspect perceptuel du fonctionnement de la prononciation variable en français. On peut affirmer que les processus de relâchement en français dérivent leur valeur en tant que marqueurs de classe sociale de la divergence bien perceptible de ces relâchements de l'orthographe française. Ainsi, l'élision du / $\mathrm{r}$ / en finale de syllabe, dans un mot tel que quatre, produit la forme [kat], alors que la réalisation canonique est [kat $\mathrm{B}]$. Cette divergence de la prononciation standard, qui reflète le $<r>$ de l'orthographe, est nettement apparente. De même, l'élision du /l/ produit une déviation de la prononciation que représente l'orthographe standard, comme par exemple dans elle parle prononcé [عрaьl] (standard [عlpasl]); ou de façon plus proéminente, dans les mots lexicaux tel que escalier réalisé

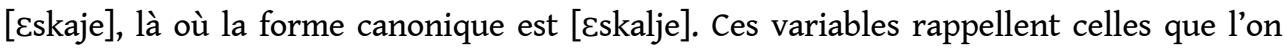
trouve en anglais ((h), (ing) et le coup de glotte) dont discutent Chambers et Trudgill (1998: 72). Les auteurs suggèrent que ces variables fassent l'objet d'une stigmatisation bien apparente en raison justement de cette "divergence entre la prononciation et l'orthographe ». Parallèlement, l'élision du schwa et de /l/ et /r/ est " naturelle » dans le sens employé ci-dessus. Mais dans un pays tel que la France, où la tradition normative est très forte, ces processus ne seront certainement pas regardés comme naturels par des locuteurs-auditeurs qui ont intériorisé cette attitude normative envers la variation langagière et qui identifient ces variables en tant que marqueurs de classe sociale. Si cette analyse est exacte, la conclusion que l'on peut en tirer est qu'un accent ouvrier est déprécié en grande partie parce qu'il véhicule une trajectoire sociale non normée.

La problématique est de savoir ce qui en français équivaut aux variables vocaliques anglaises qui, à la différence des traits dits naturels, ont un rapport indirect avec l'orthographe et véhiculent les aspects de l'identité régionale du locuteur, le plus souvent urbaine. On affirme couramment que les accents ouvriers urbains anglais sont tous hautement dépréciés; il existe cependant une hiérarchie à l'intérieur de ces accents, ou plutôt un continuum multidimensionnel comportant les qualités telles que l'humour, l'amabilité et la solidarité, ainsi que des traits négatifs. Ces informations sociales supplémentaires dérivent des stéréotypes régionaux et risquent parfois de produire des interférences qui entravent ou même vicient le message propositionnel articulé. On peut présumer que ces attributs s'expriment à travers les traits de prononciation quantifiables, au moins en partie; il est alors question de savoir si les Français usent de moyens linguistiques susceptibles de produire le même effet. Il nous faut des résultats plus directs permettant d'établir une description précise du fonctionnement socio-régional de la prononciation variable du français, qui a été insuffisamment étudiée du point de vue variationniste, au vu de son importance démographique et culturelle. 


\section{BIBLIOGRAPHIE}

ARmstrong, N. (1996) « Deletion of French /1/ : linguistic, social and stylistic factors », Journal of French Language Studies 6/1:1-21.

ARMSTRONG, N. et UNSWORTH S. (1999) « Sociolinguistic variation in southern French schwa ", Linguistics 37/1, 127-56.

Ashby, W. (1988) « Français du Canada/français de France : divergence et convergence », French Review 61/5 : 693-702.

Chambers, J. K. et Trudgill P. (1998) Dialectology, 2e édition. Cambridge : CUP.

ECKERT, P. (1989) « The whole woman : sex and gender differences in variation », Language Variation and Change 1, 245-67.

GADET, F. (1989) Le français ordinaire. Paris : Armand Colin (2e édition, 1997).

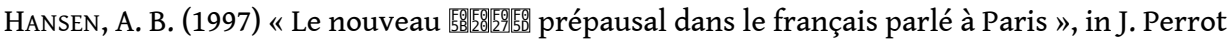
(ed.), Polyphonie pour Iván Fónagy. Mélanges offerts en hommage à Iván Fónagy par un groupe de disciples, collègues et admirateurs, 173-198. Paris : L'Harmattan.

HANSEN, A. B. (1998) Les voyelles nasales du français parisien moderne. Copenhagen : Museum Tusculanum Press.

HARMS, L. S. (1961) « Listener judgments of status cues in speech », Quarterly Journal of Speech 47, $164-68$.

HuDson, R. (1996) Sociolinguistics, 2e édition. Cambridge : CUP.

Joos, M (1961) The five clocks. New York : Harcourt.

LANDICK, M. (1995) « The mid-vowels in figures : hard facts », French Review, 69/1 : 88-103.

LAKS, B. (1983) « Langage et pratiques sociales. Etude sociolinguistique d'un groupe

d'adolescents ", Actes de la recherche en sciences sociales 46. Paris : Editions de Minuit, 73-97.

MARTINET, A. (1945) La prononciation du français contemporain. Paris : Droz.

Milroy, J., Milroy L., HARTLEY S. et WALSHAW D. (1994) « Glottal stops and Tyneside glottalisation : competing patterns of variation and change in British English", Language Variation and Change 6 : 327-357.

TRudgill, P. (1995) Sociolinguistics, 3e édition. Harmondsworth : Penguin.

WALTER, H. (1982) Enquête phonologique et variétés régionales du français. Paris : France Expansion.

WALTER, H. (1988) Le français dans tous les sens. Paris : Robert Laffont.

\section{RÉSUMÉS}

Dans cet article nous rendons compte d'une série de résultats provenant d'une expérience auditive menée à Rennes et portant sur le français de Nancy. Les résultats montrent un degré de succès élevé de la part des auditeurs rennais dans leur estimation, sur la base du seul accent, de 
la classe sociale d'un échantillon de locuteurs nancéiens. En même temps, les informateurs rennais ont fait preuve d'un manque de succès assez remarquable dans leur estimation de la provenance régionale des locuteurs nancéiens. Ces résultats indiquent que les éléments sociorégionaux des accents français socialement diagnostiqués, qui sont indissociables dans certaines langues (l'anglais notamment), ont été disloqués en France en raison des processus assez récents de standardisation et de nivellement. Ainsi, lorsqu'ils identifient les caractéristiques sociales d'un inconnu, il se peut que les auditeurs français exploitent les traits de prononciation qui relèvent principalement des phénomènes produits dans la chaîne de la langue parlée : les 'facilités de prononciation'. Ces traits de prononciation communiqueraient l'appartenance de classe sociale en France, sans pour autant indiquer l'appartenance régionale ; précisément du fait que ces traits sont partagés par toute la communauté linguistique septentrionale.

This article discusses some results relating to an identification test conducted in Rennes and focused on the speech of a sample of informants recorded in Nancy. The responses of the Rennes informants showed that they were able to identify quite successfully from their speech alone the social-class provenance of the Nancy speaker sample. However, the Rennes informants proved rather unsuccessful in identifying the regional provenance of the Nancy sample. This suggests that the social-regional components of socially diagnostic accents, which are indissociable in some languages (notably English), have been disrupted in French as a consequence of fairly recent processes of standardisation and levelling. When identifying the social characteristics of a previously unknown speaker, French listeners may therefore use pronunciation cues that are largely allegro-speech phenomena, unlocalised and therefore opaque as to regional provenance.

\section{AUTEURS}

\section{ARMSTRONG NIGEL}

Université de Leeds

Department of French Studies

Leeds LS2 9JT

Grande-Bretagne

BOUGHTON ZOË

Université de Newcastle Upon Tyne

Department of French Studies

Newcastle Upon Tyne NE1 7RU

Grande-Bretagne

z.c.boughton@ncl.ac.uk 